\title{
Disease-related phenotypes in a Drosophila model of hereditary spastic paraplegia are ameliorated by treatment with vinblastine
}

\author{
Genny Orso, ${ }^{1}$ Andrea Martinuzzi,2 Maria Giovanna Rossetto,, ${ }^{1,2}$ Elena Sartori, 1,2 \\ Mel Feany, ${ }^{3}$ and Andrea Daga ${ }^{1,2}$
}

1Dulbecco Telethon Institute and Department of Pharmacology, University of Padova, Padova, Italy. ${ }^{2} \mathrm{E}$. Medea Scientific Institute, Conegliano Research Center, Conegliano, Italy. ${ }^{3}$ Department of Pathology, Brigham and Women's Hospital and Harvard Medical School, Boston, Massachusetts, USA.

\begin{abstract}
Hereditary spastic paraplegias (HSPs) are a group of neurodegenerative diseases characterized by progressive weakness and spasticity of the lower limbs. Dominant mutations in the human SPG4 gene, encoding spastin, are responsible for the most frequent form of HSP. Spastin is an ATPase that binds microtubules and localizes to the spindle pole and distal axon in mammalian cell lines. Furthermore, its Drosophila homolog, Drosophila spastin (Dspastin), has been recently shown to regulate microtubule stability and synaptic function at the Drosophila larval neuromuscular junction. Here we report the generation of a spastin-linked HSP animal model and show that in Drosophila, neural knockdown of Dspastin and, conversely, neural overexpression of Dspastin containing a conserved pathogenic mutation both recapitulate some phenotypic aspects of the human disease, including adult onset, locomotor impairment, and neurodegeneration. At the subcellular level, neuronal expression of both Dspastin RNA interference and mutant Dspastin cause an excessive stabilization of microtubules in the neuromuscular junction synapse. In addition, we provide evidence that administration of the microtubule targeting drug vinblastine significantly attenuates these phenotypes in vivo. Our findings demonstrate that loss of spastin function elicits HSP-like phenotypes in Drosophila, provide novel insights into the molecular mechanism of spastin mutations, and raise the possibility that therapy with Vinca alkaloids may be efficacious in spastin-associated HSP and other disorders related to microtubule dysfunction.
\end{abstract}

\section{Introduction}

Hereditary spastic paraplegias (HSPs) are a group of neurodegenerative diseases characterized by progressive weakness and spasticity of the lower limbs (1-4). They are conventionally subdivided into pure and complicated forms, depending on the absence or presence of additional symptoms (2). Pure HSP is the single largest group of HSP pathologies, and the principal histopathological feature of disease appears to be a length-dependent retrograde degeneration of the terminal ends of axons of the corticospinal tracts and fasciculi gracilis (1-4), even though new evidence is emerging of neuropathologic changes outside the motor system $(5,6)$. Dominant mutations in the human SPG4 gene, encoding spastin, are responsible for the prevailing form of pure HSP (7). A wide variety of nonsense, missense, and frameshift mutations have been identified in SPG4 patients and produce clinically indistinguishable phenotypes, suggesting that the molecular mechanism of spastin mutations is haploinsufficiency $(8,9)$. Nevertheless, cellular expression of pathogenic missense mutations potentially inactivating the ATPase domain of spastin has led to the hypothesis that truncated or missense mutant spastin may cause HSP through a dominant-negative mechanism $(10,11)$.

SPG4 encodes spastin, a protein belonging to large AAA ATPase family characterized by a conserved domain contain-

Nonstandard abbreviations used: Dspastin, Drosophila spastin; GMR, glass multiple reporter; HSP, hereditary spastic paraplegia; NMJ, neuromuscular junction; RNAi, RNA interference; UAS, upstream activating sequence.

Conflict of interest: The authors have declared that no conflict of interest exists.

Citation for this article: J. Clin. Invest. 115:3026-3034 (2005).

doi:10.1172/JCI24694. ing Walker A and B ATP-binding motifs. Spastin N-terminal region contains the MIT domain, a recently identified protein motif found in microtubule-interacting proteins (12). Indeed, human spastin has been shown to bind microtubules in an ATPdependent fashion (10), suggesting a function in microtubule severing, as was shown for its close relative katanin p60, which is the catalytic subunit of a microtubule-severing protein (13). Experimental evidence that both human and Drosophila spastin proteins display ATPase activity and use energy from ATP hydrolysis to sever and disassemble microtubules in vitro has been reported very recently $(14,15)$.

At this stage, intracellular localization of spastin remains controversial, and both nuclear and cytoplasmic localization has been reported $(6,10,11,16)$. More recently, spastin has been found to be enriched in cell regions containing dynamic microtubules, such as the spindle pole and the distal axon (17).

Robust evidence supporting a role for spastin in microtubule cytoskeleton dynamics in vivo has come from studies in Drosophila. Drosophila has been shown to contain a highly conserved spastin homolog, Drosophila spastin (Dspastin) (18). In contrast to previous reports that mammalian spastin has a nuclear localization, Dspastin was demonstrated to be a cytoplasmic protein enriched in neuronal axons and synaptic connections. At the neuromuscular junction (NMJ) synapse, loss of Dspastin causes morphological undergrowth and loss of synaptic area. Moreover, using antibodies specific for posttranslationally modified tubulin found exclusively in stabilized microtubules, it has been found that Dspastin regulates microtubule stability at the NMJ synapse: Dspastin overexpression decreases microtubule stability, whereas loss of Dspastin enhances microtubule stability (19). Very recently, 

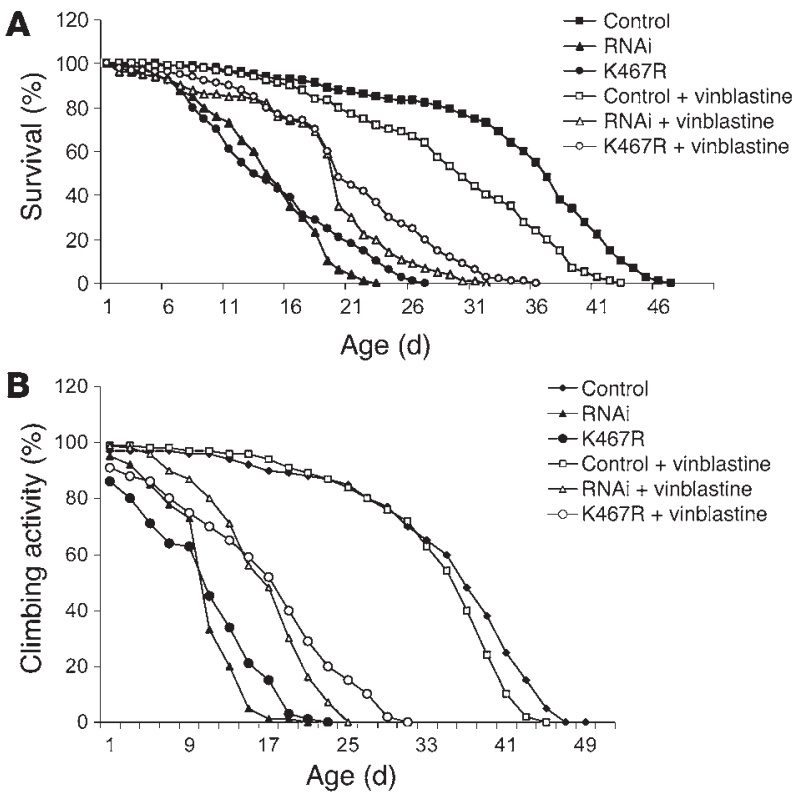

another study on Dspastin has reported a function for this protein in the regulation of synaptic microtubule networks (20). These in vivo data in Drosophila strongly support previous in vitro data in other systems, providing a likely cause for the neuronal dysfunction in spastin-associated HSP disease.

Here we report the generation of the first animal model to our knowledge for spastin-linked HSP and show that in Drosophila, neural knockdown of Dspastin and, conversely, neural overexpression of Dspastin containing a conserved pathogenic mutation both recapitulate some phenotypic aspects of the human disease. Moreover, we provide evidence that these phenotypes can be significantly relieved in vivo by the microtubule targeting drug vinblastine. Our findings demonstrate that loss of spastin function elicits HSP-like phenotypes in Drosophila, provide evidence that at least a subset of spastin mutations may produce disease via a dominant-negative mechanism, and raise the possibility that therapy with Vinca alkaloids may be efficacious in spastin-associated HSP and other disorders related to microtubule dysfunction.

\section{Figure 1}

Behavioral phenotypes caused by neuron-specific expression of Dspastin RNAi and the Dspastin K467R pathogenic mutant are efficiently antagonized by pharmacological treatment with vinblastine. (A and B) Neuronal transgenic expression of Dspastin RNAi and Dspastin K467R reduced the longevity of adult flies $(\mathbf{A})$ and severely impaired adult locomotor performance (B), causing premature loss of climbing ability. Vinblastine treatment increased adult lifespan (A) and improved locomotor ability (B), as judged by the enhanced performance in the climbing assay, of flies expressing Dspastin RNAi and Dspastin K467R in neurons. Control genotypes were as follows: Elav-Gal4/+, UAS-Dspastin-RNAi/+, and UAS-Dspastin-K467R/+. Experimental genotypes were as follows: Elav-Gal4/+;UAS-Dspastin-RNAi/+ and Elav-Gal4/+;UAS-Dspastin-K467R/+.

\section{Results}

Neuron-specific knockdown of Dspastin results in HSP-related phenotypes. It is unclear how mutations in the spastin gene give rise to HSP in humans. However, the dominant inheritance pattern and broad mutational spectrum indicate that the molecular pathogenetic mechanism is likely to be partial loss of spastin function, determined by haploinsufficiency of the SPG4 locus to which some neurons are acutely susceptible $(1-4,9)$. The spastin protein is highly enriched within the nervous systems of mammals, although not exclusively confined to neurons (16). Similarly, recent work has demonstrated that Dspastin message levels are elevated in the nervous system during Drosophila embryonic development (18), and that the Dspastin protein is greatly enriched in larval neurons (19). As in mammals, expression of Dspastin is not restricted to the Drosophila nervous system, being easily detectable, for example, in the larval musculature.

We therefore sought to determine whether specific neuronal downregulation of Dspastin in Drosophila might produce phenotypes reminiscent of the human pathology. Although a Dspastin loss-of-function mutant is now available, only very few escapers survive, and these die very shortly after eclosion, making adult-onset phenotypes due to neuronal dysfunction impossible to evaluate. We thus employed tissue-specific RNA interference-mediated (RNAi-mediated) knockdown of Dspastin, using upstream activating sequence-Dspastin-RNAi (UAS-Dspastin-RNAi) transgenic lines highly efficient in reducing Dspastin mRNA and protein levels (19). To further confirm the efficacy

\section{Figure 2}

Reducing Dspastin expression produces age-dependent neurodegeneration. (A) One-day-old fly with transgenic expression of Dspastin RNAi showed no sign of neurodegeneration. (B) Twenty-day-old fly with transgenic expression of Dspastin RNAi had numerous vacuoles in the cortex and neuropil (arrows). (C) Twenty-day-old control fly showed no evidence of neurodegeneration. (D) Degenerating cells were TUNEL positive (arrows). (E) No TUNEL-positive cells were present in age-matched control flies. Control genotypes were as follows: Elav-Gal4/+ and UAS-Dspastin-RNAi/+. The experimental genotype used was Elav-Gal4/+;UAS-Dspastin-RNAi/+.
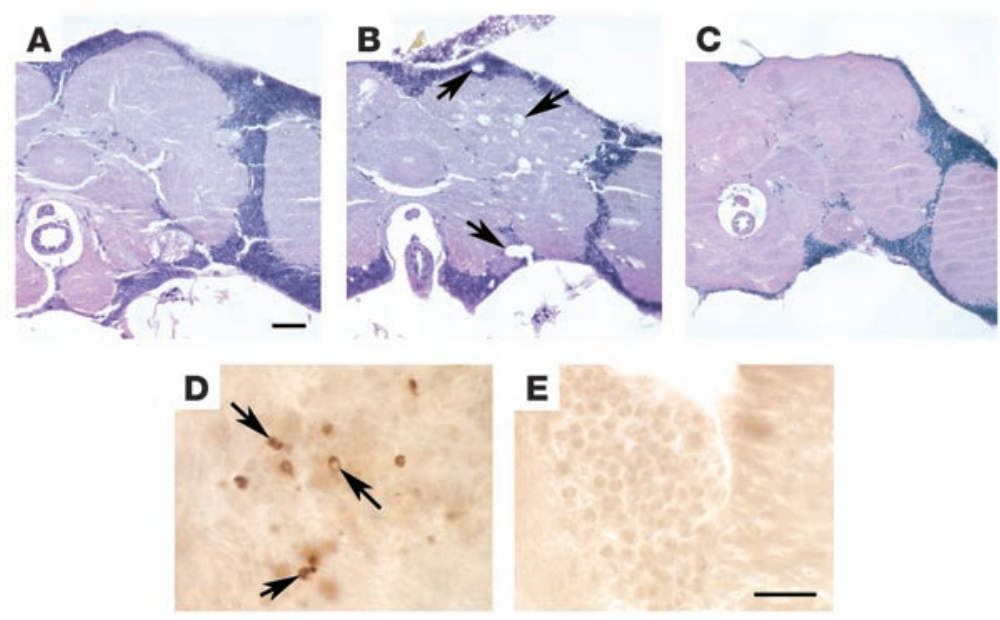


\section{Table 1}

Summary of the phenotypic effects of tissue-specific expression of different Dspastin transgenes
Transgene
Dspastin RNAi

Dspastin wild-type

\section{Ubiquitous}

Pupa lethal with escapers $(20 \%)$

Pupa lethal with escapers $(4 \%)$ Embryo lethal

\section{Expression pattern} Nervous system

Viable, short lifespan, motor dysfunction, neurodegeneration

Viable, short lifespan, motor dysfunction Embryo lethal

\section{Eye}

Normal

Normal

Small, rough eye
To achieve the desired pattern of expression, the following Gal4 driver lines were used: tubulin-Gal4 for ubiquitous expression, Elav-Gal4 for nervous system expression, and GMR-Gal4 for eye expression. Percentages given indicate the percentage of escapers.

locomotor performance declined more rapidly than that of controls (Figure 1B). The reduced climbing response of Dspastin RNAi flies demonstrates that decreased Dspastin dosage in the nervous system causes a significant, progressive motor deficit that is reminiscent of an important feature of HSP and further substantiates a functional conservation between Drosophila and human spastin proteins.

To establish whether these behavioral phenotypes were associated with neurodegeneration, we assessed fly brain morphology by examining histologic sections and assaying for apoptotic cells by TUNEL staining. The brain of newly eclosed Dspastin RNAi flies showed normal anatomical and histological organization (Figure 2A). In contrast, aged Dspastin RNAi flies displayed numerous vacuoles in the neuropil and cortex (Figure 2B, arrows), a hallmark

of our UAS-Dspastin-RNAi transgene, we obtained the Dspastin 5.75 allele, a recently described null Dspastin mutant (20), and performed a detailed phenotypic comparison between the ubiquitous expression of Dspastin-RNAi and Dspastin ${ }^{5.75}$ mutants. These comparative analyses demonstrate that Dspastin ${ }^{5.75}$ mutants display behavioral (i.e., eclosion rates) and cytological defects (i.e., decrease of synaptic area and accumulation of stable microtubules at the NMJ synapse) entirely overlapping with those produced by global expression of Dspastin RNAi (Supplemental Figure 3; supplemental material available online with this article; doi:10.1172/JCI24694DS1). Our observations demonstrate that RNAi-mediated knockdown of Dspastin accurately phenocopies the absence of Dspastin protein.

Expression of UAS-Dspastin-RNAi can be targeted to a specific tissue using the heterologous Gal4/UAS binary expression system (21). Use of this system allows for exclusive tissue-specific decrease of Dspastin levels, thus overcoming potential pleiotropic effects due to genetic mutation of Dspastin. Expression of UAS-DspastinRNAi in a pan-neuronal pattern using the Elav-Gal4 driver did not result in lethality prior to eclosion, permitting a detailed analysis of adult-onset phenotypes induced by loss of Dspastin activity. Newly eclosed Elav-Gal4/+;UAS-Dspastin-RNAi/+ flies appeared normal as revealed by viability, morphology, and behavior. However, as time progressed, reduction of Dspastin in these animals resulted in a marked shortening of adult lifespan (Figure 1A). Although lifespan is apparently not affected in HSP patients, this result suggests that knockdown of Dspastin in Drosophila gradually compromises adult nervous system functionality, which may in turn contribute to early lethality.

The principal clinical feature of HSP is progressive spasticity and weakness of the lower limbs, eventually leading to a dramatic motor deficit. To ascertain whether flies with reduced Dspastin expression exhibit similar progressive motor dysfunction, we used the climbing assay. Normal Drosophila display a strong negative geotactic response. When tapped to the bottom of a vial they rapidly rise to the top of the vial, and most individuals remain there. As they get older or manifest locomotor dysfunction, normal flies no longer climb to the top of the vial, but instead make short, abortive climbs and fall back to the bottom of the vial. This assay was previously identified as a reliable measure of locomotor performance in Drosophila (22-25). When tested for their climbing ability, Elav-Gal4/+;UAS-Dspastin-RNAi/+ young adults displayed a normal climbing response. However, over time, their of neurodegeneration in Drosophila (26-28). Vacuoles were not present in sections from age-matched control flies (Figure 2C). TUNEL-positive cells were present in the cortex of aged Dspastin RNAi flies (Figure 2D), but not in control flies (Figure 2E). These findings are consistent with adult-onset neurodegeneration mediated by reduced levels of Dspastin. These results strongly suggest a causal relationship between the locomotor deficit and shortened lifespan caused by loss of Dspastin function in the Drosophila adult nervous system and age-dependent brain degeneration.

Mutant Dspastin behaves as a dominant negative in vivo. While nonsense, frameshift, or splice-site mutations in human spastin have
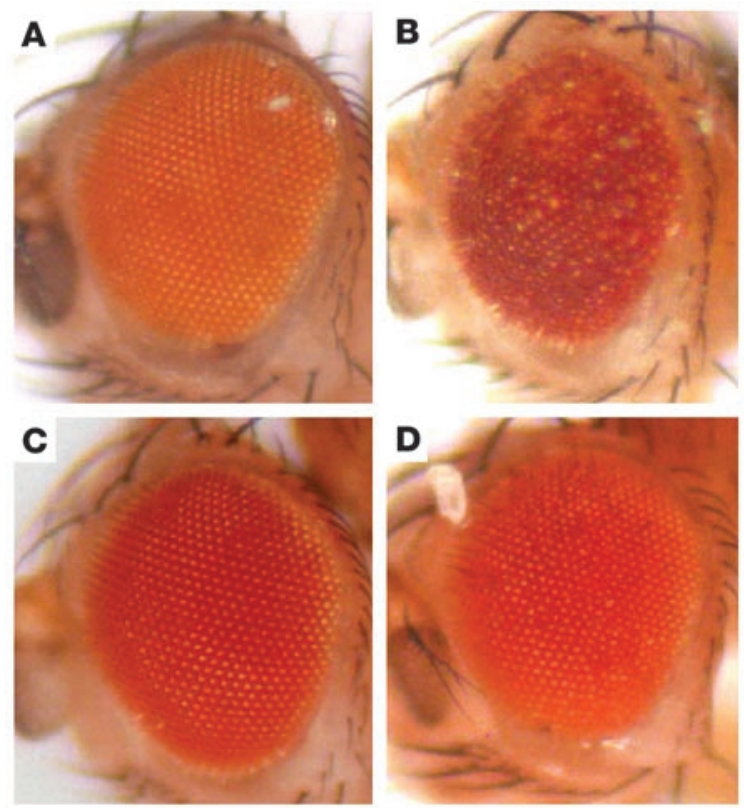

\section{Figure 3}

Expression of Dspastin K467R in the Drosophila eye opposes the phenotypic effects of wild-type Dspastin expression. (A) Wild-type Drosophila eye. (B) Eyes expressing low levels of UAS-Dspastin under the control of GMR-Gal4 displayed a moderate rough phenotype. (C) Expression of UAS-Dspastin-K467R alone had no phenotypic consequences in the eye. (D) Simultaneous expression of both constructs resulted in a practically normal external morphology, suggesting that Dspastin K467R suppresses the rough eye phenotype caused by UAS-Dspastin expression. 
A
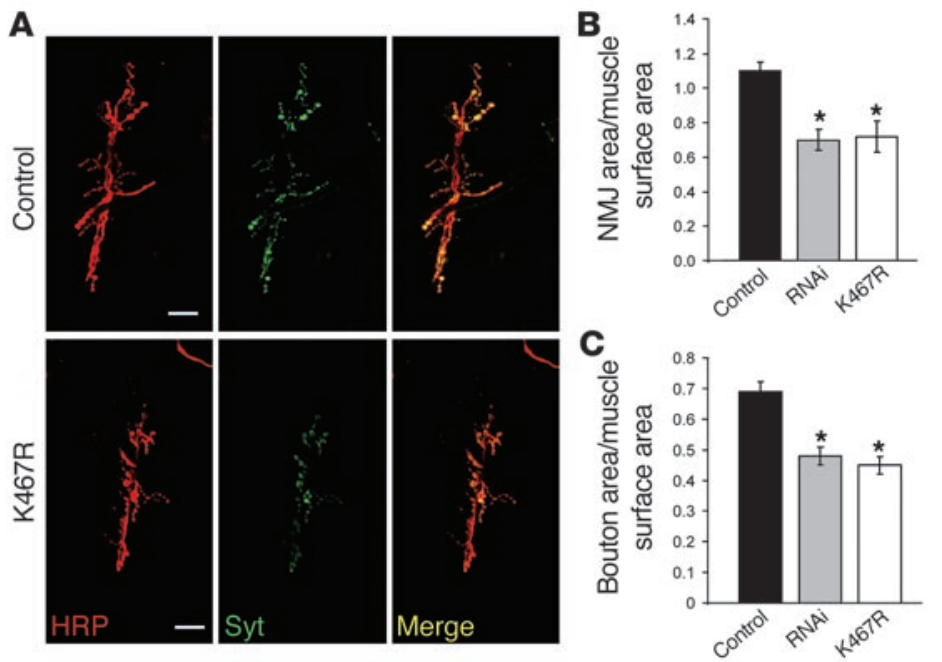

\section{Figure 4}

Neuronal expression of pathological mutant Dspastin K467R and Dspastin RNAi affect synaptic morphology in a comparable fashion. (A) Larval NMJ at muscles $6 / 7$, identified by HRP immunocytochemistry (red; left panels). Neural expression of Dspastin K467R in a wild-type background caused a reduction of the synaptic area and arborization compared with controls (Elav-Gal4/+ and UAS-Dspastin-K467R/+). NMJs were also labeled with anti-synaptotagmin antibody (Syt) to provide an alternative means of measuring synaptic area (green; middle panels). Merged images are shown at right. Scale bars: approximately $20 \mu \mathrm{M}$. (B and C) Quantification of the data using results of both HRP $(\mathbf{B})$ and synaptotagmin $(\mathbf{C})$ staining shows that the observed decrease in synaptic area was statistically significant and similar to that detected in NMJs expressing Dspastin RNAi. ${ }^{*} P<0.0001$. suggested a haploinsufficiency mechanism for the human disorder, cellular expression of pathogenic missense mutations potentially inactivating the ATPase domain of spastin has led to the hypothesis that the disease may also be caused by a dominant-negative mechanism. To address the pathogenetic mechanism underlying spastin mutations, we generated transgenic flies bearing a K467R substitution that corresponded to pathogenic mutation K388R in the human spastin protein. We chose this mutation because in mammalian cell lines, spastin K388R has been shown to bind microtubules constitutively (10), and analogous amino acid changes in ATPases belonging to the same family are known to abrogate ATP binding and elicit dominant-negative effects $(29,30)$. To examine the functional significance of the K467R mutant, we expressed it in a wild-type background (i.e., in flies with normal amounts of Dspastin), a genetic combination closely resembling that of SPG4 patients, where wild-type and mutated spastin are simultaneously present. We reasoned that a dominant-negative mutant should give rise to phenotypes similar to those produced by downregulation of Dspastin but distinct from those caused by Dspastin overexpression. Consistent with this hypothesis, eye-specific expression of both UAS-Dspastin-RNAi and UAS-Dspastin-K467R had no phenotypic effects, while expression of UAS-Dspastin gave rise to external eye roughness (Table 1 and Figure 3, B and C), suggesting that replacement of lysine 467 with arginine leads to inactivation of the protein. Moreover, similar to the expression of UAS-Dspastin-RNAi, ubiquitous and nervous system-specific expression of UAS-Dspastin-K467R resulted in partial lethality at the pupa stage and shortening of adult lifespan, respectively. In contrast, UAS-Dspastin driven ubiquitously by tubulin-Gal4, or expressed in a neuronal pattern by Elav-Gal4, consistently caused early embryonic lethality (Table 1). Immunohistochemical analyses using an anti-HRP antibody to highlight the larval NMJ synapse, an anti-synaptotagmin antibody to label exclusively synaptic boutons, and an anti-acetylated $\alpha$-tubulin antibody to specifically identify stabilized microtubules revealed that expression of the K467R mutation in wild-type neurons resulted in a decrease in synaptic area (Figure 4) as well as the accumulation of hyperstable microtubules at the NMJ presynaptic terminal (Figure 5) as was previously demonstrated for neuronal Dspastin knockdown (19), suggesting that these synaptic defects are produced by loss of endogenous Dspastin activity mediated by expression of Dspastin K467R.
We next assessed adult vitality, climbing response, brain morphology, and extent of neuronal apoptosis in Elav-Gal4/+;UASDspastin-K467R/+ flies. Reduction of lifespan and impairment of locomotor activity were remarkably similar in Dspastin RNAi and K467R mutant flies (Figure 1, A and B). Although progressive neurodegeneration in K467R-expressing brains was modest (Supplemental Figure 2B), when K467R was expressed in the presence of reduced levels of Dspastin (Elav-Gal4/+;UAS-Dspastin-K467R/+; Dspastin $5.75 /+)$, significant degeneration was seen, particularly in the medulla (Supplemental Figure 2C). The observation that knockdown of Dspastin and overexpression of the Dspastin K467R mutant consistently elicited similar phenotypes suggests that the K467R mutation acts through a dominant-negative mechanism by interfering with the function of endogenous Dspastin.

Corroboration for a dominant-negative role of the K467R mutation has also come from a comparison of the phenotype caused by co-overexpression of Dspastin and Dspastin K467R with those caused by overexpression of each alone. In the Drosophila eye, moderate ectopic expression of Dspastin produced a mild rough eye phenotype, whereas overexpression of Dspastin K467R had no phenotypic consequences (Figure 3, B and C), indicating that this pathologic amino acid substitution causes loss of spastin protein activity. However, simultaneous expression of both transgenes resulted in essentially normal external eye morphology, strongly suggesting that the K467R mutant causes suppression of the rough eye induced by ectopic expression of Dspastin (Figure 3D). Taken together, our findings demonstrate that in Drosophila overexpression of Dspastin K467R and Dspastin RNAi cause largely overlapping phenotypes reminiscent of HSP pathology, and that pathogenic mutation K467R acts as a dominant negative.

Administration of vinblastine attenuates disease-related phenotypes in vivo. Dspastin has been recently shown to play a role in destabilizing the microtubule cytoskeleton in neurons. Loss of this Dspastin activity leads to the accumulation of excessively stable microtubules in the larval NMJ, thereby affecting synaptic neurotransmission (19). Synaptic function, however, can be restored by treating Dspastin RNAi dissected larval preparation with nocodazole (19). This observation prompted us to investigate whether pharmacological treatment targeted at microtubules could counteract in vivo the phenotypic effects of expressing Dspastin RNAi and Dspastin K467R. Feeding has been previously shown to be an efficient means of administering 
A
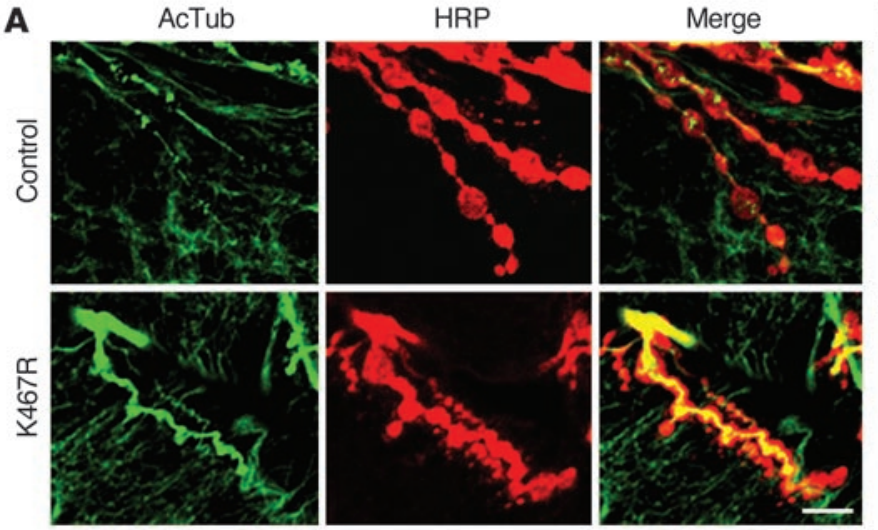

B

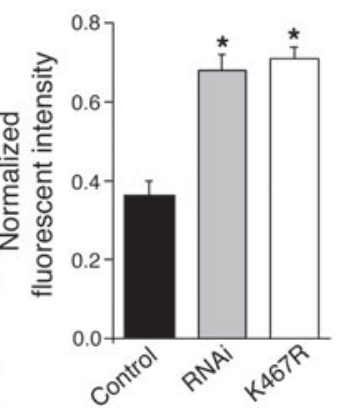

\section{Figure 5}

Neuronal expression of pathological mutant Dspastin K467R and Dspastin RNAi induce an analogous hyperstabilization of microtubules. (A) Images of NMJ immunocytochemistry using an anti-HRP antibody (red; middle panels), a neuronal maker of the presynaptic arbor, and an anti-acetylated $\alpha$-tubulin antibody (AcTub, green; left panels), present only in stable microtubules (33), demonstrate that nervous system expression of Dspastin K467R induced accumulation of stabilized microtubules, as indicated by the dramatic increase of acetylated $\alpha$-tubulin signal compared with controls (ElavGal4/+ and UAS-Dspastin-K467R/+). (B) Quantification of the data in A revealed that this increase was statistically significant and comparable to that caused by RNAi knockdown of Dspastin. Values were normalized as described in Methods. ${ }^{\star} P<0.0001$. Scale bar: approximately $10 \mu \mathrm{M}$. the NMJ presynaptic terminal (Figure $7, \mathrm{~A}-\mathrm{C})$ and the increase in acetylated $\alpha$-tubulin (Figure 8) of flies expressing Dspastin RNAi and Dspastin K467R in the nervous system, thus specifically rescuing the cytological defect caused by loss of Dspastin activity (Figures 4 and 5). We also tested the ability of vinblastine to suppress the NMJ phenotypes observed in Dspastin ${ }^{5.75}$-null mutants. In these individuals, synaptic boutons are more numerous, and synaptic area is reduced compared with controls (Supplemental Figure 3). However, administration of vinblastine produced a moderate but statistically significant increase in total synaptic area and a decrease in bouton number (Figure 7, D-G), suggesting that this drug is capable of ameliorating the phenotypes associated with loss of Dspastin function in the complete absence of spastin protein from neuronal as well as muscle cells. drugs to flies (31-35). Thus, we monitored the effects of feeding flies with nocodazole and vinblastine, 2 structurally and mechanistically diverse microtubule destabilizing drugs $(36,37)$. These drugs were assayed for their ability to suppress both the lethality induced by ubiquitous expression of Dspastin RNAi and Dspastin K467R and the motor and adult lifespan phenotypes observed in Elav-Gal4/+;UASDspastin-RNAi/+ and Elav-Gal4/+;UAS-Dspastin-K467R/+ flies. Surprisingly, in vivo treatment with nocodazole did not rescue any of the above phenotypes (data not shown). In contrast, administration of vinblastine resulted in a suppression of both Dspastin RNAi- and Dspastin K467R-induced phenotypes. Global expression of Dspastin RNAi and Dspastin K467R normally results in lethality with a few escapers; however, treatment with vinblastine led to a substantial increase in the eclosion rate of flies ubiquitously expressing both RNAi and the K467R mutant (Figure 6). Similarly, a greater proportion of Dspastin ${ }^{5.75}$-null mutant Drosophila eclosed in food containing vinblastine than in food lacking this drug (Figure 6), even though the effect of vinblastine on Dspastin ${ }^{5.75}$ mutants appears to be less pronounced than that on tubulin-Gal4/+;UAS-Dspastin-RNAi/+ individuals, possibly because the positive effects of vinblastine were aided by a residual presence of Dspastin protein in RNAi-treated flies. Consistent with these observations, vinblastine-fed Elav-Gal4/+; UAS-Dspastin-RNAi/+ and Elav-Gal4/+;UAS-Dspastin-K467R/+ Drosophila displayed a moderate but statistically significant improvement in both locomotor ability and adult lifespan compared to untreated controls $(P<0.05$; Figure 1$)$. It is to be noted, however, that vinblastine treatment was associated with small levels of toxicity (Figure 1 and Supplemental Figure 4). We then determined whether suppression of these behavioral phenotypes correlated at the cellular level with arrest of neuronal degeneration and rescue of synaptic area reduction as well as aberrant microtubule accumulation at the larval NMJ. While administration of vinblastine did not rescue terminal neurodegeneration of Elav-Gal4/+;UAS-Dspastin$\mathrm{RNAi} /+$, it was remarkably capable of reversing the reduced area of

\section{Discussion}

The most common form of HSP, accounting for approximately $40 \%$ of all cases, is caused by mutations in the SPG4 gene, which encodes the spastin protein. To date, no vertebrate or invertebrate animal models for spastin-linked HSP have been developed to allow a better understanding of the basic pathophysiologic mechanisms responsible for this disease. Although human spastin is widely expressed, the nervous system appears to be specifically vulnerable to the partial loss of spastin protein observed in SPG4 patients. Likewise, Dspastin expression is not restricted to the nervous system, indicating that an analysis of neuronal specific phenotypes due to loss of Dspastin may be hampered by pleiotropic effects caused by the lack of Dspastin in other tissues. We have

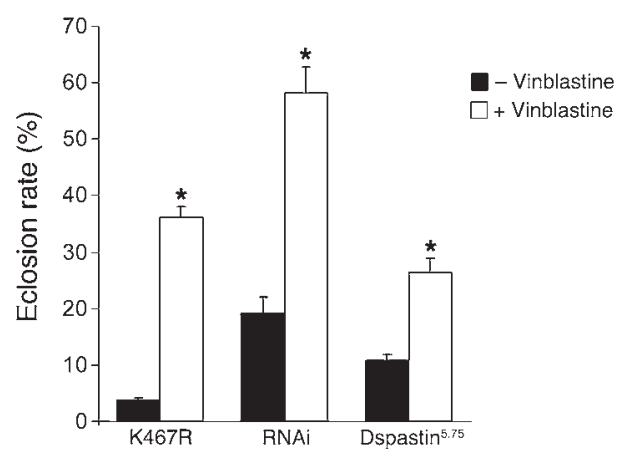

\section{Figure 6}

In vivo consequences of vinblastine administration. Addition of vinblastine to the food significantly increased the eclosion rate of $D$ spastin ${ }^{5.75}$ null mutants and that of flies ubiquitously expressing Dspastin RNAi and Dspastin K467R compared with genotypically identical flies raised in the absence of vinblastine. Experimental genotypes were as follows: tubulin-Gal4/+;UAS-Dspastin-RNAi/+, tubulin-Gal4/+;UAS-DspastinK467R/+, and Dspastin 5.75/Dspastin ${ }^{5.75} .{ }^{*} P<0.0001$. 


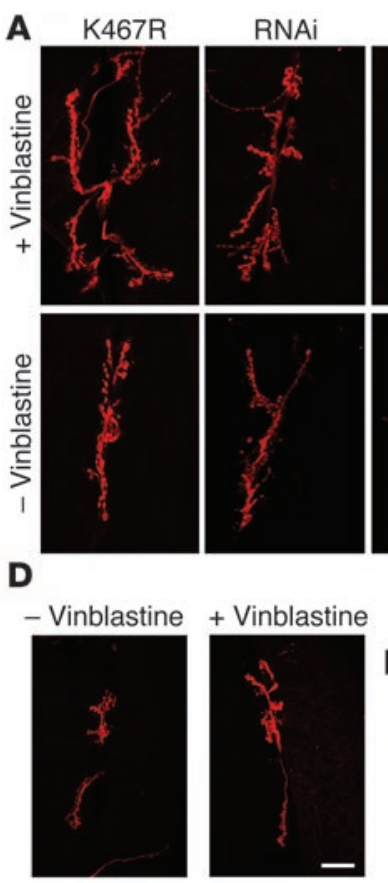

Figure 7

Administration of vinblastine reverts the loss of synaptic area caused by nervous system-specific expression of Dspastin RNAi and Dspastin K467R and ameliorates NMJ phenotypes of Dspastin 5.75 mutants. (A and D) Representative images of NMJ synapses at muscles 6/7 revealed with antiHRP antibody (red) show that vinblastine treatment partially restored synaptic area in Elav-Gal4/+; UAS-Dspastin-RNAi/+ and Elav-Gal4/+;UAS-Dspastin-K467R/+ (A) and Dspastin ${ }^{5.75}$ Drosophila (D). Control genotypes were as follows: Elav-Gal4/+, UAS-Dspastin-RNAi/+, and UAS-DspastinK467R/+. Scale bars: approximately $20 \mu \mathrm{M}$. (B, C, E, and F) Quantification of synaptic area restoration, determined both by anti-HRP (B and $\mathbf{E})$ and anti-synaptotagmin $(\mathbf{C}$ and $\mathbf{F})$ immunoreactivity, shows that phenotypic rescue by vinblastine was statistically significant. (G) Vinblastine treatment produced a statistically significant decrease in bouton number of Dspastin ${ }^{5.75}$ third instar larva NMJ. ${ }^{\star \star} P<0.05 ;{ }^{\star} P<0.0001$.

therefore employed a tissue-specific transgenic RNAi strategy for the selective elimination of Dspastin from the fly nervous system in order to create a spastin-linked HSP disease model in Drosophila that allowed us to examine the phenotypic consequences of neuron-specific removal of Dspastin.

Recently, a study has been published describing phenotypes for Dspastin-null mutant flies that contrast with our present data (20). For instance, the authors report that in Dspastin-null mutants, there are fewer microtubule bundles within the NMJ, in contrast to the accumulation of hyperstabilized microtubules that we observed at the NMJ upon expression of Dspastin RNAi. A direct comparison of our results with those presented by Sherwood et al. is not possible, because we have knocked down Dspastin specifically in the nervous system, while the null allele used by Sherwood et al. affects all tissues in which Dspastin is normally expressed (20). However, to rectify these apparent discrepancies, we have carried out a detailed comparative analysis between Dspastin ${ }^{5.75}$-null mutant Drosophila and Drosophila expressing Dspastin RNAi ubiquitously (see Supplemental Discussion). We found that eclosion rates were similar between Dspastin ${ }^{5.75}$ and Dspastin RNAi flies, that synaptic area was similarly decreased, and that the levels of acetylated $\alpha$-tubulin were analogously increased in synaptic terminals of Dspastin 5.75 and Dspastin RNAi flies (Supplemental Figure 3).
Interestingly, Drosophila lacking Dspastin exclusively within their nervous systems displayed mutant phenotypes that resembled observations made in HSP patients. First, there was no lethality associated with neuronal loss of Dspastin activity, and flies eclosed into apparently normal young adults. Indeed, HSP is not a lethal condition, and patients present variable ages of onset, indicating that there are no obvious developmental defects linked to spastin mutations or, if present, these defects become manifest only after birth. Second, as time advanced, these flies progressively developed severe movement defects and within around 15-20 days of birth stopped moving altogether. Progressive locomotor dysfunction and impaired gait are the hallmark of HSP pathology. In addition to these disease-related abnormalities, a marked shortening of adult lifespan was also associated with loss of Dspastin in Drosophila that is not observed in SPG4 patients. Third, HSP is thought to be a progressive neurodegenerative disorder. Consistent with this notion, nervous system-specific reduction of Dspastin function resulted in progressive neurodegeneration of the fly brain with obvious presence of neurons undergoing apoptosis. Although in SPG4 patients the most prominent neuropathological finding is a progressive retrograde degeneration of corticospinal tracts without obvious loss of anterior horn cells, new evidence is emerging that neuronal involvement in SPG4 HSP is not always limited to distal axons of long spinal cord neurons, but may be 

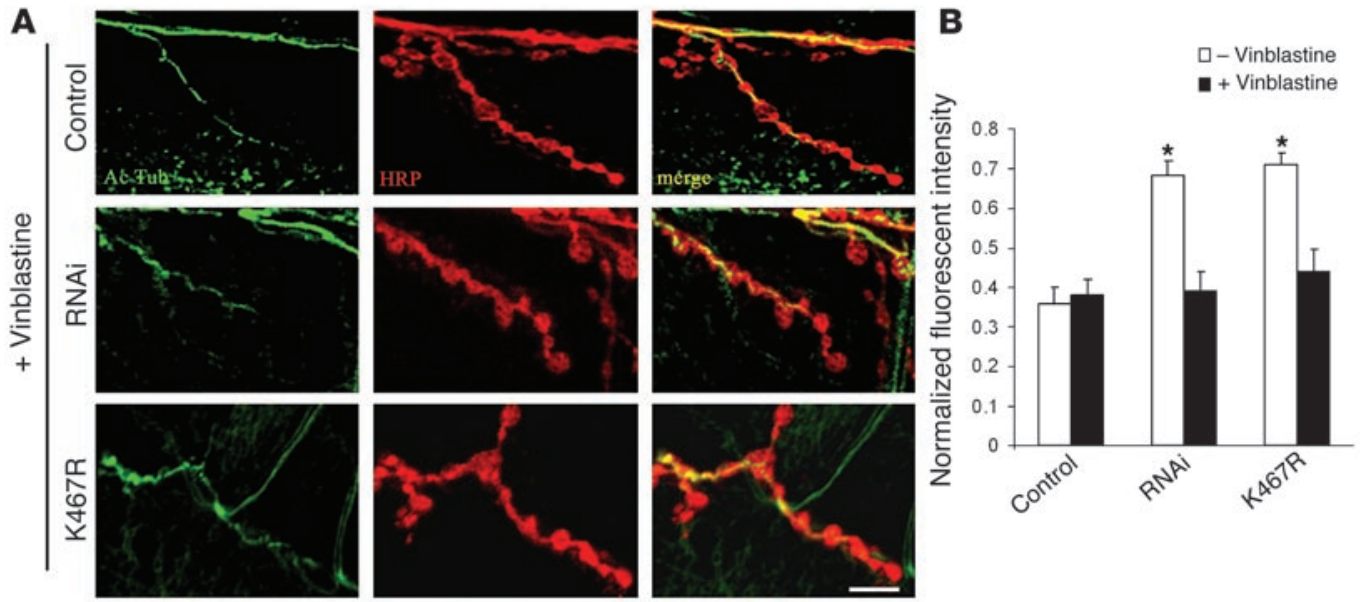

Figure 8

Administration of vinblastine suppresses the accumulation of stabilized microtubules caused by nervous system-specific expression of $D$ spastin RNAi and Dspastin K467R. (A) Confocal images of NMJ synaptic terminals labeled with the neuronal marker anti-HRP antibody (red) and an anti-acetylated $\alpha$-tubulin antibody (green), a marker for stable microtubules (33), demonstrate that in vinblastine-treated Elav-Gal4/+;UASDspastin-RNAi/+ and Elav-Gal4/+;UAS-Dspastin-K467R/+ individuals, acetylated $\alpha$-tubulin levels were reduced compared with those of untreated animals and similar to those of controls raised in vinblastine. Control genotypes were as follows: Elav-Gal4/+, UAS-Dspastin-RNAi/+, and UAS-Dspastin-K467R/+. Scale bar: approximately $10 \mu \mathrm{M}$. (B) Quantification of the decrease of acetylated $\alpha$-tubulin signal shown in A demonstrates that phenotypic rescue by vinblastine was statistically significant. ${ }^{*} P<0.0001$.

more widespread and affect neurons outside the motor system (5, $6)$. An alternative explanation for the greater severity of the phenotypes observed in flies is that the neurodegeneration and cell loss seen in our Drosophila model may reflect the dramatic reduction of Dspastin levels we achieved through our RNAi-knockdown approach compared with the more moderate partial loss of spastin function thought to underlie HSP.

The mechanisms by which spastin mutations produce dominant HSP in humans are controversial. The broad mutational spectrum observed has suggested that the molecular mechanism is likely to be haploinsufficiency; however, it has also been proposed that truncated or missense mutant spastin may function as a dominant negative. The outstanding sequence homology between human spastin and Dspastin proteins has allowed us to use our model system to investigate the mode of action of pathogenic spastin mutations. Our results demonstrate that neuronally targeted expression of the Dspastin K467R mutant (corresponding to pathologic mutation K388R in humans) in a fly containing wild-type levels of endogenous Dspastin caused behavioral phenotypes that largely overlapped with those produced by the specific removal of Dspastin from neurons. These phenotypes include adult-onset progressive locomotor impairment and neurodegeneration in the context of reductions in Dspastin levels. Likewise, we provide evidence that at the molecular level, neuron-specific knockdown of Dspastin and neuron-specific expression of the Dspastin K467R mutant induced an analogous reduction of synaptic area and accumulation of hyperstabilized microtubule cytoskeleton at the neuromuscular synapse. The observation that loss of Dspastin activity and overexpression of a pathogenic Dspastin mutant caused analogous phenotypic consequences strongly suggests that in both instances, the observed abnormalities are mediated by loss of endogenous Dspastin activity, and thus at least some disease mutations act through a dominant-negative mechanism. These results further suggest that aberrant microtubule stabilization in neurons is likely to be the underlying cause of SPG4 HSP in humans.
Since human spastin containing the K388R substitution (corresponding to the K467R mutation in Drosophila) has been shown to bind microtubules as effectively as wild-type spastin, the simplest mechanistic explanation for the dominant-negative effect of Dspastin K467R is that spastin binding sites on microtubules are titrated out by the inactive mutant, thus preventing the action of wild-type spastin. This explanation may also account for the low levels of neurodegeneration observed in Dspastin K467R brains compared with Dspastin RNAi brains. While expression of Dspastin RNAi dramatically reduces the levels of endogenous Dspastin, its function is likely to be only partially blocked by overexpression of the dominant-negative K467R mutant. In neurons expressing Dspastin K467R, greater residual Dspastin function could result in histologically more subtle, but physiologically important, phenotypes. This interpretation is supported by the observation that expression of the K467R mutant in a Dspastin ${ }^{5.75}$-null heterozygous background, where the levels of endogenous Dspastin are reduced by $50 \%$, resulted in obvious degeneration.

The dominant-negative pathogenic mechanism of the K467R mutant could be identical for missense and other mutations that may alter the function of the ATPase domain of spastin, leaving its functional microtubule binding region intact. However, pathogenic frameshift mutations have been identified that result in truncation of the spastin protein upstream of the microtubulebinding domain $(38,39)$. Therefore, spastin mutations may cause HSP pathology by 2 distinct mechanisms: loss of wild-type spastin function mediated by a dominant-negative effect or decrease in spastin dosage through haploinsufficiency (16).

The generation of a disease model with features reminiscent of the human disease and whose biochemical and cytological defects are well characterized has allowed us to focus on the identification of pharmacological agents capable of suppressing the phenotypes induced by loss of Dspastin function in vivo, whether direct or mediated by the expression of the Dspastin K467R mutant. Using this approach, we have shown that in Drosophila the microtubule 
destabilizing agent vinblastine, but not nocodazole (another potential candidate drug), significantly attenuated disease-related abnormalities in vivo, and this attenuation correlated with a robust suppression of the cytological defects of the neuromuscular synapse cytoskeleton associated with these abnormalities. At low concentrations, vinblastine is thought to exert its action by modulating microtubule dynamics rather than by decreasing tubulin polymer mass (40). Therefore, our findings imply that vinblastine treatment rescues the pathological effects of loss of Dspastin function in vivo by pharmacologically compensating for an increase in microtubule stability caused by downregulation or complete loss of Dspastin and further confirm that misregulation of microtubule dynamics is likely responsible for the pathogenesis of SPG4. Since no pharmacological treatment is currently available for this disorder and vinblastine, as well as several other Vinca alkaloid family members, are currently widely used clinically in cancer chemotherapy, these drugs could be rapidly tested as potential therapeutic agents for spastin-linked HSP and related diseases.

In summary, we have created the first SPG4 animal model to our knowledge and shown that in Drosophila, RNAi-mediated knockdown of Dspastin and overexpression of the Dspastin K467R pathogenic mutant reproduce salient features of the human disorder: adult onset, progressive motor dysfunction, and progressive neurodegeneration. Our Drosophila model demonstrates that a dominant-negative mechanism is implicated in the pathogenesis of spastin-associated HSP and provides compelling evidence that pharmacological modulation of microtubule stability in vivo effectively counters the pathological phenotypes arising from loss of Dspastin function, strongly supporting the hypothesis that alteration of microtubule dynamics underlies the human disease.

Despite the apparent parallels underscored by the data presented, Drosophila can mimic the human disease only in part due to the obviously much simpler anatomical and physiological organization of flies. However, existing data indicate that the underlying biochemical and cytological defects, both in mammalian cells and in flies, are traceable to the misregulation of microtubule stability in nerve terminals caused by loss of spastin function.

Almost half of all HSP disease cases are linked to microtubule motor proteins or other proteins with microtubule interacting domains (e.g., KIF5A, spartin, and spastin) $(12,41,42)$. The availability of a genetically tractable animal model for spastin-associated HSP may lead to new insights into this group of diseases by helping to identify common pathways through which alterations in microtubule cytoskeleton formation, stability, and/or function can cause different forms of HSP.

\section{Methods}

Drosophila stocks and crosses. The UAS-Dspastin, UAS-Dspastin-RNAi, and Dspastin ${ }^{5.75}$-null Drosophila used in this study were described previously $(19,20)$. Site-directed mutagenesis was employed to generate the UASDspastin-K467R construct. Nine independent transgenic lines were derived for the K467R mutant, and all were tested for protein expression by immunohistochemistry using different Gal4 driver lines. The 2 lines with the highest expression levels, which consequently displayed the most severe phenotypes, were chosen for the studies reported here.

The Gal4 activator lines used in this study were Elav-Gal4, tubulin-Gal4, and glass multiple reporter-Gal4 (GMR-Gal4; Bloomington Stock Center, Indiana University). To increase protein and double-stranded RNA expression, all experimental crosses were performed at $28^{\circ} \mathrm{C}$.
Histology. Heads from adult flies were fixed in formalin and embedded in paraffin, and $4-\mu \mathrm{m}$ frontal sections were prepared. Serial sections were cut through the entire brain and placed on a single glass slide. Hematoxylin and eosin staining was performed using a standard protocol. Apoptosis was detected with the TUNEL assay using a commercially available kit (TdT FragEl; Oncogene).

Immunobistochemistry. NMJ immunostaining was performed on wandering third instar larvae reared at $28^{\circ} \mathrm{C}$. Larvae were dissected in standard saline and fixed in $4 \%$ paraformaldehyde for 45 minutes. Preparations were washed in PBS containing 0.5\% bovine serum and labeled overnight with anti-HRP antibody (1:1000; Sigma-Aldrich), anti-synaptotagmin (1:100; Developmental Studies Hybridoma Bank, University of Iowa), and antiacetylated $\alpha$-tubulin (1:1000; Sigma-Aldrich). Secondary antibodies were from Invitrogen Corp. Third instar larva NMJs on abdominal muscle 6/7 from thoracic segment 2 were assayed for total synaptic area, highlighting areas of HRP and synaptotagmin immunoreactivity. Total synaptic area, determined either by anti-HRP or anti-synaptotagmin staining, was normalized to the surface area of the corresponding muscle. Quantification of immunohistochemistry was achieved by normalizing the intensity of the acetylated $\alpha$-tubulin signal to that of HRP, as previously described (19). At least 10 individual NMJs were analyzed for each experiment. Images were acquired with a Nikon $\mathrm{C} 1$ confocal microscope and analyzed using either Nikon EZ-C1 (version 2.10) or NIH ImageJ (version 1.32J) software.

Adult behavioral analysis. Adult climbing test was used to measure Drosophila locomotor function. For each genotype tested, 30 individual flies, maintained at $28^{\circ} \mathrm{C}$, were collected and placed into an empty vial with a line drawn 2 centimeters from the bottom of the tube. After a 1-hour recovery period from anesthesia, flies were gently tapped to the bottom of the tube, and the number of flies that successfully climbed above the 2 -cm mark after 20 seconds was noted. Fifteen separate and consecutive trials were performed, and the results were averaged. At least 300 flies were tested for each genotype. Lifespan experiments were performed starting with 300-400 animals for each genotype. Flies were collected at 1 day after eclosion and placed in vials containing 50 animals. The animals were maintained at $28^{\circ} \mathrm{C}$, transferred to fresh medium every 2 days, and the number of dead flies was counted. Adult climbing and lifespan experiments were repeated at least 3 times.

Vinblastine treatment. Vinblastine sulfate (Sigma-Aldrich) was added to regular Drosophila food, and concentrations ranging from $0.01 \mu \mathrm{M}$ to $1 \mu \mathrm{M}$ were tested. To perform the experiments presented in this study, we selected the lowest effective concentration $(0.05 \mu \mathrm{M})$.

Statistics. Statistical analyses were performed using unpaired 2-tailed Student's $t$ tests and were carried out using Prism (version 3.03; GraphPad) or Microsoft Excel software.

\section{Acknowledgments}

We are grateful to Kai Zinn for providing the Dspastin ${ }^{5.75}$ Drosophila line. We thank Tim Lebetsky for helpful discussions, Davide Patergnani for technical support, and Erica Zanarella and Diana Pendin for help with tedious fly work. This study was supported by grants from the Telethon Foundation (TCP00059; to A. Daga) and Compagnia San Paolo (S00059CSP; to A. Daga).

Received for publication February 9, 2005, and accepted in revised form August 23, 2005.

Address correspondence to: Andrea Daga, Dulbecco Telethon Institute and Department of Pharmacology, Largo Meneghetti 2, University of Padova, Padova 35131, Italy. Phone: 39-0498275778; Fax: 39-0498275093; E-mail: daga@unipd.it. 
1. Reid, E. 2003. Science in motion: common molecular pathological themes emerge in the hereditary spastic paraplegias. J. Med. Genet. 40:81-86.

2. Harding, A.E. 1983. Classification of the hereditary ataxias and paraplegias. Lancet. 1:1151-1155.

3. Fink, J.K. 2003. The hereditary spastic paraplegias: nine genes and counting. Arch. Neurol. 60:1045-1049

4. Fink, J.K. 2003. Advances in the hereditary spastic paraplegias. Exp. Neurol. 184(Suppl. 1):S106-S110.

5. Wharton, S.B., et al. 2003. The cellular and molecular pathology of the motor system in hereditary spastic paraparesis due to mutation of the spastin gene. J. Neuropathol. Exp. Neurol. 62:1166-1177.

6. Orlacchio, A., et al. 2004. Hereditary spastic paraplegia: clinical genetic study of 15 families. Arch. Neurol. 61:849-855.

7. Hazan, J., et al. 1999. Spastin, a new AAA protein, is altered in the most frequent form of autosomal dominant spastic paraplegia. Nat. Genet. 23:296-303.

8. Lindsey, J.C., et al. 2000. Mutation analysis of the spastin gene (SPG4) in patients with hereditary spastic paraparesis. J. Med. Genet. 37:759-765.

9. Fonknechten, N., et al. 2000. Spectrum of SPG4 mutations in autosomal dominant spastic paraplegia. Hum. Mol. Genet. 9:637-644.

10. Errico, A., Ballabio, A., and Rugarli, E.I. 2002. Spastin, the protein mutated in autosomal dominant hereditary spastic paraplegia, is involved in microtubule dynamics. Hum. Mol. Genet. 11:153-163.

11. McDermott, C.J., et al. 2003. Hereditary spastic paraparesis: disrupted intracellular transport associated with spastin mutation. Ann. Neurol. 54:748-759.

12. Ciccarelli, F.D., et al. 2003. The identification of a conserved domain in both spartin and spastin, mutated in hereditary spastic paraplegia. Genomics. 81:437-441

13. McNally, F.J., and Vale, R.D. 1993. Identification of katanin, an ATPase that severs and disassembles stable microtubules. Cell. 75:419-429.

14. Evans, K.J., Gomes, E.R., Reisenweber, S.M., Gundersen, G.G., and Lauring, B.P. 2005. Linking axonal degeneration to microtubule remodeling by Spastin-mediated microtubule severing. J. Cell Biol. 168:599-606

15. Roll-Mecak, A., and Vale, R.D. 2005. The Drosophila homologue of the hereditary spastic paraplegia protein, spastin, severs and disassembles microtubules. Curr. Biol. 15:650-655.
16. Charvin, D., et al. 2003. Mutations of SPG4 are responsible for a loss of function of spastin, an abundant neuronal protein localized in the nucleus. Hum. Mol. Genet. 12:71-78.

17. Errico, A., Claudiani, P., D’Addio, M., and Rugarli, E.I. 2004. Spastin interacts with the centrosomal protein NA14, and is enriched in the spindle pole, the midbody and the distal axon. Hum. Mol. Genet. 13:2121-2132

18. Kammermeier, L., Spring, J., Stierwald, M., Burgunder, J.M., and Reichert, H. 2003. Identification of the Drosophila melanogaster homolog of the human spastin gene. Dev. Genes Evol. 213:412-415.

19. Trotta, N., Orso, G., Rossetto, M.G., Daga, A., and Broadie, K. 2004. The hereditary spastic paraplegia gene, spastin, regulates microtubule stability to modulate synaptic structure and function. Curr. Biol. 14:1135-1147.

20. Sherwood, N.T., Sun, Q., Xue, M., Zhang, B., and Zinn, K. 2004. Drosophila spastin regulates synaptic microtubule networks and is required for normal motor function. PLoS Biol. 2:e429.

21. Brand, A.H., and Perrimon, N. 1993. Targeted gene expression as a means of altering cell fates and generating dominant phenotypes. Development. 118:401-415

22. Ganetzky, B., and Flanagan, J.R. 1978. On the relationship between senescence and age-related changes in two wild-type strains of Drosophila melanogaster. Exp. Gerontol. 13:189-196.

23. Le Bourg, E., and Lints, F.A. 1992. Hypergravity and aging in Drosophila melanogaster. 4. Climbing activity. Gerontology. 38:59-64.

24. Feany, M.B., and Bender, W.W. 2000. A Drosophila model of Parkinson's disease. Nature. 404:394-398.

25. Deleault, N.R., et al. 2003. Post-transcriptional suppression of pathogenic prion protein expression in Drosophila neurons. J. Neurochem. 85:1614-1623.

26. Buchanan, R.L., and Benzer, S. 1993. Defective glia in the Drosophila brain degeneration mutant drop-dead. Neuron. 10:839-850.

27. Kretzschmar, D., Hasan, G., Sharma, S., Heisenberg, M., and Benzer, S. 1997. The swiss cheese mutant causes glial hyperwrapping and brain degeneration in Drosophila. J. Neurosci. 17:7425-7432.

28. Wittmann, C.W., et al. 2001. Tauopathy in Drosophila: neurodegeneration without neurofibrillary tangles. Science. 293:711-714.

29. Babst, M., Wendland, B., Estepa, E.J., and Emr, S.D.
1998. The Vps4p AAA ATPase regulates membrane association of a Vps protein complex required for normal endosome function. EMBOJ. 17:2982-2993.

30. McNally, K.P., Bazirgan, O.A., and McNally, F.J. 2000. Two domains of $\mathrm{p} 80$ katanin regulate microtubule severing and spindle pole targeting by $\mathrm{p} 60$ katanin. J. Cell Sci. 113:1623-1633.

31. Min, K.T., and Benzer, S. 1999. Preventing neurodegeneration in the Drosophila mutant bubblegum. Science. 284:1985-1988.

32. Kang, H.L., Benzer, S., and Min, K.T. 2002. Life extension in Drosophila by feeding a drug. Proc. Natl. Acad. Sci. U. S. A. 99:838-843.

33. Steffan, J.S., et al. 2001. Histone deacetylase inhibitors arrest polyglutamine-dependent neurodegeneration in Drosophila. Nature. 413:739-743.

34. Auluck, P.K., and Bonini, N.M. 2002. Pharmacological prevention of Parkinson disease in Drosophila. Nat. Med. 8:1185-1186.

35. Marsh, J.L., and Thompson, L.M. 2004. Can flies help humans treat neurodegenerative diseases? Bioessays. 26:485-496.

36. Jordan, M.A., Thrower, D., and Wilson, L. 1992. Effects of vinblastine, podophyllotoxin and nocodazole on mitotic spindles. Implications for the role of microtubule dynamics in mitosis. J. Cell Sci. 102:401-416.

37. Dhamodharan, R., Jordan, M.A., Thrower, D., Wilson, L., and Wadsworth, P. 1995. Vinblastine suppresses dynamics of individual microtubules in living interphase cells. Mol. Biol. Cell. 6:1215-1229.

38. Proukakis, C., Hart, P.E., Cornish, A., Warner, T.T., and Crosby, A.H. 2002. Three novel spastin (SPG4) mutations in families with autosomal dominant hereditary spastic paraplegia. J. Neurol. Sci. 201:65-69.

39. Sauter, S., et al. 2002. Mutation analysis of the spastin gene (SPG4) in patients in Germany with autosomal dominant hereditary spastic paraplegia. Hum. Mutat. 20:127-132.

40. Jordan, M.A., and Wilson, L. 2004. Microtubules as a target for anticancer drugs. Nat. Rev. Cancer. 4:253-265.

41. Patel, H., et al. 2002. SPG20 is mutated in Troyer syndrome, an hereditary spastic paraplegia. Nat. Genet. 31:347-348.

42. Reid, E., et al. 2002. A kinesin heavy chain (KIF5A) mutation in hereditary spastic paraplegia (SPG10). Am.J. Hum. Genet. 71:1189-1194. 